\title{
Sistemas de Cultivo no Cerrado e Dinâmica de Populações de Plantas Daninhas ${ }^{1}$
}

\author{
Tillage Systems in the Cerrado and Dinamics of Weed Populations
}

PEREIRA, F.A.R. ${ }^{2}$ e VELINI, E.D. ${ }^{3}$

\begin{abstract}
RESUMO - O trabalho teve como objetivo avaliar os efeitos de sistemas de plantio direto, utilizando diferentes programas de rotação de culturas, de preparo convencional e de cultivo mínimo sobre a dinâmica de populações de plantas daninhas nas condições edafoclimáticas do cerrado. Utilizou-se o delineamento experimental de blocos ao acaso, com quatro repetições e cinco tratamentos, sendo dois de plantio direto que constaram de programas de rotação constituídos por culturas de verão e de outono/inverno e dois em monocultivo, nos sistemas de preparo convencional e cultivo mínimo respectivamente. Avaliou-se a dinâmica das populações de plantas daninhas. A maior eficiência de controle cultural da comunidade infestante foi obtida com o sistema de plantio direto, por meio dos seguintes programas de rotação: (sorgo/soja - crotalária/milho - milheto/soja - milho safrinha/soja); (milho safrinha/ soja - girassol/milho - sorgo/soja - girassol/milho) e (guandu/soja - milheto/soja - milho safrinha/milho - girassol/soja).
\end{abstract}

Palavras-chave: rotação de culturas.

\begin{abstract}
This research aimed to evaluate the effects of no-tillage systems using crop rotation variations, conventional tillage, and minimum tillage on the dynamics of weed populations in the cerrado region in Brazil. The experiment was arranged in a randomized block design, four replications and five treatments, (two no tillage treatments consisting of summer and fall/ winter crop rotations and two single crop treatments under conventional tillage and minimum tillage. Weed population dynamics was evaluated. The results showed that the no-tillage system was the most efficient for weed control with the following programs: soybean/sorghum Crotalaria/corn - millet/soybean, winter corn/soybean); (winter corn/soybean - sunflower/ corn - sorghum/soybean - sunflower/corn) and (Pigeon/pea/soybean-millet/soybean - winter corn/corn - sunflower/soybean) .
\end{abstract}

Key words: crop rotation.

\section{INTRODUÇÃO}

A região dos cerrados apresenta peculiaridades ambientais que a tornam distinta dos demais ambientes agrícolas do Brasil. Assim, nem sempre uma tecnologia preconizada em outras regiões pode ser empregada no cerrado, mesmo tratando-se de uma cultura comum.
Portanto, as recomendações técnicas devem ser baseadas em resultados de pesquisas desenvolvidas nas condições edafoclimáticas locais.

O plantio direto nos cerrados já é considerado uma técnica consagrada, embora muitos fatores técnicos ainda necessitem de ajustes. Dentre as dificuldades, destacam-se

1 Recebido para publicação em 1.4.2003 e na forma revisada em 12.12.2003.

Parte da Tese de Doutorado em Agricultura, FCA-UNESP Botucatu, SP 18603-970.

2 Eng.-Agr. Prof. Dr. - UNIDERP, Programa de Pós-Graduação em Produção e Gestão Agroindustrial, Campus III, Rua Alexandre Herculano, 1400, 79037-280 Campo Grande-MS; ${ }^{3}$ Eng.-Agr. Prof. Dr. - FCA-UNESP, Caixa Postal 237, $18603-970$ Botucatu-SP. 
as poucas opções de espécies vegetais para a formação da cobertura morta e o desconhecimento de alternativas de programas de rotação de culturas que proporcionem a otimização do sistema. De acordo com Ruedell (1995), o controle de plantas daninhas é considerado o item de maior dificuldade a ser dominado pelos usuários do plantio direto, comparativamente à mecanização e à fertilização. O controle químico, apesar de ser uma forma eficiente de eliminar as plantas daninhas, se usado de maneira inadequada, pode onerar o custo de produção e/ou não apresentar eficácia. O uso integrado do plantio direto e da rotação de culturas pode resultar na redução das infestações de plantas daninhas ao longo dos anos. A rotação de culturas no inverno e no verão possibilita o emprego de técnicas diferenciadas de controle de plantas daninhas e o uso de herbicidas com eficiência e mecanismos de ação diferenciados, diminuindo os riscos com falhas de controle e o desenvolvimento do processo da resistência de plantas daninhas a herbicidas (Bianchi, 1998).

A escolha de um programa de rotação de culturas, entre outros fatores, poderá também definir o comportamento evolutivo da flora infestante da área. Em pesquisas realizadas por Almeida (1985), no Estado do Paraná, verificouse que diferentes programas de rotação de culturas causaram efeitos diferenciados sobre a caracterização da comunidade infestante do terreno, indicando que, dependendo das culturas em rotação e da respectiva combinação cronológica de cultivo, a dinâmica das plantas daninhas pode sofrer alterações mais ou menos significativas.

O presente trabalho foi desenvolvido com o objetivo de avaliar diferentes programas de rotação de culturas, num solo sob cerrado, em sistemas de plantio direto, cultivo mínimo e convencional, quanto à eficiência na redução da infestação por plantas daninhas.

\section{MATERIAL E MÉTODOS}

O experimento foi desenvolvido na Fazenda Elo, localizada no município de Chapadão do Céu, GO; o solo da área experimental é um Latossolo Vermelho distroférrico (LVdf), A moderado, textura argilosa, fase campo cerrado e relevo plano.

Planta Daninha, Viçosa-MG, v.21, n.3, p.355-363, 2003
O delineamento experimental empregado foi o de blocos ao acaso, com quatro repetições e parcelas de $10,0 \times 50,0 \mathrm{~m}$, com área útil de $8,0 \times 40,0 \mathrm{~m}$. Os tratamentos, em número de sete, foram compostos por cinco programas de rotação de culturas em sistema de plantio direto, um programa de monocultivo em plantio convencional e um programa de monocultivo em cultivo mínimo.

A pesquisa foi desenvolvida durante quatro anos, no período de 1994/95 a 1997/98. Os programas de rotação de culturas eram compostos de culturas de verão (safra) representativas da região e de culturas de outono/ inverno (entressafra), com o objetivo de manter o solo coberto e fornecer massa vegetal para formar a cobertura morta, condição imprescindivel para a semeadura direta.

Na Tabela 1 são apresentados os tratamentos com as respectivas combinações dos cultivos em cada programa de rotação de culturas.

O preparo convencional constituiu-se de uma gradagem pesada e duas a três gradagens leves. No cultivo mínimo, utilizou-se como fonte de cobertura morta a massa vegetal formada pela comunidade infestante.

Avaliou-se a dinâmica da flora infestante, identificando as plantas daninhas ocorrentes em cada tratamento, procedeu-se à contagem e obtiveram-se as suas respectivas densidades populacionais. As avaliações foram realizadas imediatamente antes do manejo químico das culturas de outono/inverno e aos 20 a 30 dias após o plantio das culturas de verão, coincidindo com a época de controle das plantas daninhas em pós-emergência. Para coleta dos dados, utilizou-se um quadro amostrador de $0,5 \times 0,5 \mathrm{~m}$, que era lançado aleatoriamente em dez pontos distribuídos em zigue zague na área útil das parcelas.

Os resultados das densidades das comunidades infestantes foram submetidos à análise de variância, sendo as médias comparadas pelo teste de Tukey a $5 \%$ de probabilidade.

\section{RESULTADOS E DISCUSSÃO}

Durante o período estudado, foram identificadas 16 espécies de plantas daninhas, distribuídas em 10 famílias, conforme 
Tabela 1 - Descrição dos tratamentos e seqüência cronológica dos cultivos

\begin{tabular}{|c|c|c|c|c|c|c|}
\hline \multirow{2}{*}{ Tratamento } & \multirow{2}{*}{$\begin{array}{l}\text { Sistema de } \\
\text { Cultivo }\end{array}$} & \multirow{2}{*}{$\begin{array}{l}\text { Programa de } \\
\text { Rotação }\end{array}$} & $1^{0}$ ano & $2^{0}$ ano & $3^{0}$ ano & $4^{0}$ ano \\
\hline & & & \multicolumn{4}{|c|}{ Inverno/Verão } \\
\hline 1 & PD & A & $\mathrm{Ms} / \mathrm{S}$ & $\mathrm{Gi} / \mathrm{M}$ & So/S & $\mathrm{Gi} / \mathrm{M}$ \\
\hline 2 & PD & B & $\mathrm{So} / \mathrm{S}$ & $\mathrm{CR} / \mathrm{M}$ & $\mathrm{Mt} / \mathrm{S}$ & Ms/S \\
\hline 3 & $\mathrm{PD}$ & $\mathrm{C}$ & $\mathrm{G} / \mathrm{S}$ & $\mathrm{Mt} / \mathrm{Sp}$ & $\mathrm{Ms} / \mathrm{M}$ & $\mathrm{Gi} / \mathrm{S}$ \\
\hline 4 & $\mathrm{PD}$ & D & $\mathrm{Fe} / \mathrm{Mp}$ & $\mathrm{Fe}-\mathrm{Mt} / \mathrm{S}$ & $\mathrm{AV} / \mathrm{S}$ & $\mathrm{So} / \mathrm{S}$ \\
\hline 5 & PD & E & $\mathrm{Mt} / \mathrm{S}$ & $\mathrm{G} / \mathrm{Mp}$ & $\mathrm{Fe}-\mathrm{Mt} / \mathrm{S}$ & $\mathrm{Mt} / \mathrm{M}$ \\
\hline 6 & PC & $\mathrm{F}$ & $\mathrm{Pc} / \mathrm{S}$ & $\mathrm{Pc} / \mathrm{S}$ & $\mathrm{Pc} / \mathrm{M}$ & $\mathrm{Pc} / \mathrm{S}$ \\
\hline 7 & $\mathrm{CM}$ & G & $\mathrm{Po} / \mathrm{S}$ & $\mathrm{Po} / \mathrm{S}$ & $\mathrm{Po} / \mathrm{M}$ & $\mathrm{Po} / \mathrm{S}$ \\
\hline
\end{tabular}

$\mathrm{PD}=$ plantio direto, $\mathrm{PC}=$ plantio convencional, $\mathrm{CM}=$ cultivo mínimo, $\mathrm{Mt}=$ milheto, $\mathrm{Sp}=$ soja precoce, $\mathrm{M}=$ milho, $\mathrm{Fe}=$ feijão, $\mathrm{Mp}=$ milho precoce, $\mathrm{AV}=$ aveia, $\mathrm{Ms}=$ milho safrinha, $\mathrm{So}=$ sorgo, $\mathrm{G}=$ guandu, $\mathrm{Gi}=$ girassol, $\mathrm{CR}=$ crotalaria, $\mathrm{Pc}=$ preparo convencional, $\mathrm{S}=$ soja e Po = pousio (cultivo mínimo).

apresentado na Tabela 2. As espécies mais importantes na região estavam presentes na área experimental.

Os resultados dos levantamentos das plantas daninhas nos quatro anos da pesquisa revelaram que a quantidade de espécies daninhas identificadas foi maior nas avaliações da primeira época (no manejo) do que nas de segunda (pós-emergência). A possível justificativa é que, na ocasião do manejo químico das coberturas, já haviam ocorrido as primeiras chuvas do ano agrícola, desencadeando-se o processo de germinação do banco de sementes, resultando na emergência de plântulas.

Em todos os anos, o maior número de espécies foi encontrado no tratamento 7 (cultivo mínimo). Blanco et al. (1994), verificando a distribuição mensal de emergência de plantas daninhas, constataram que, em média, 70\% da emergência das plântulas resulta do primeiro fluxo de germinação das sementes, no mês de outubro.

Dentre as espécies, Bidens pilosa esteve presente em todos os tratamentos nos quatro anos, caracterizada como planta de maior freqüência e de ocorrência estável, independentemente das alterações de cultivo ou da rotação de culturas.

A espécie Ipomoea grandifolia apresentouse também com elevada freqüência no primeiro ano; no entanto, com o decorrer dos cultivos sua densidade populacional foi reduzida. No quarto ano a presença de IAOGR só foi verificada no tratamento 2 , com uma densidade média de 3 plantas $\mathrm{m}^{2}$.
Tabela 2 - Identificação das plantas daninhas avaliadas no período de 1994/95 a 1997/98, de acordo com Lorenzi (2000)

\begin{tabular}{|c|c|c|}
\hline Família & Espécie/Nome Comum & Código \\
\hline \multirow{2}{*}{ Amaranthaceae } & $\begin{array}{l}\text { Alternanthera tenella Colla } \\
\text { (apaga-fogo) }\end{array}$ & ALRTE \\
\hline & $\begin{array}{l}\text { Amaranthus viridis L. } \\
\text { (caruru-verde) }\end{array}$ & AMAVI \\
\hline Commelineaceae & $\begin{array}{c}\text { Commelina benghalensis } \mathrm{L} . \\
\text { (trapoeraba) }\end{array}$ & COMBE \\
\hline \multirow{2}{*}{$\begin{array}{l}\text { Compositae } \\
\text { (Asteraceae) }\end{array}$} & $\begin{array}{c}\text { Ageratum conyzoides } \mathrm{L} . \\
\text { (mentrasto) }\end{array}$ & AGECO \\
\hline & $\begin{array}{l}\text { Bidens pilosa } \mathrm{L} . \\
\text { (picão-preto) }\end{array}$ & BIDPI \\
\hline Convolvulaceae & $\begin{array}{l}\text { Ipomoea grandifolia (Dammer) } \\
\text { O'Donell } \\
\quad \text { (corda-de-viola) } \\
\end{array}$ & IAOGR \\
\hline \multirow{2}{*}{ Euphorbiaceae } & $\begin{array}{c}\text { Euphorbia heterophylla } \mathrm{L} . \\
\text { (leiteiro) }\end{array}$ & EPHHL \\
\hline & $\begin{array}{c}\text { Chamaesyce hirta (L.) Millsp. } \\
\text { (erva-de-santa-luzia) }\end{array}$ & ЕPHHI \\
\hline \multirow{4}{*}{$\begin{array}{l}\text { Gramineae } \\
\text { (Poaceae) }\end{array}$} & $\begin{array}{l}\text { Cenchrus echinatus } \mathrm{L} . \\
\text { (capim-carrapicho) }\end{array}$ & CCHEC \\
\hline & $\begin{array}{l}\text { Digitaria horizontalis Willd. } \\
\text { (capim-colchão) }\end{array}$ & DIGHO \\
\hline & $\begin{array}{l}\text { Brachiaria plantaginea (Link) } \\
\text { Hitchc. } \\
\text { (capim-marmelada) }\end{array}$ & BRAPL \\
\hline & $\begin{array}{c}\text { Digitaria insularis (L.) Fedde } \\
\text { (capim-amargoso) }\end{array}$ & DIGIN \\
\hline Labitae & $\begin{array}{l}\text { Leonotis nepetifolia }(\mathrm{L} .) \\
\text { W.T.Aiton } \\
\qquad \text { (cordão-de-frade) }\end{array}$ & LEONE \\
\hline Malvaceae & $\begin{array}{l}\text { Sida rhombifolia } \mathrm{L} . \\
\text { (guanxuma) }\end{array}$ & SIDRH \\
\hline Portulacaceae & $\begin{array}{c}\text { Portulaca oleraceae } \mathrm{L} . \\
\text { (beldroega) }\end{array}$ & POROL \\
\hline Rubiaceae & $\begin{array}{c}\text { Spermacoce latifolia Aubl. } \\
\text { (erva-quente) }\end{array}$ & BOILF \\
\hline
\end{tabular}


A espécie Euphorbia heterophylla esteve presente em todos os anos no tratamento 7 (cultivo mínimo); nos três primeiros anos, nos tratamentos 1 e 5; e, no quarto ano, nos tratamentos 2, 3 e 7 . A dinâmica de EPHHL já foi estudada em regiões com uso freqüente de plantio direto no Estado do Paraná, constatando-se a seleção desta espécie em áreas de plantio direto (Adegas, 1998). Em pesquisa realizada por Vargas et al. (1999), comprovouse que a taxa de germinação das sementes de EPHHL foi significativamente reduzida com o aumento da profundidade no solo, isto é, as sementes na superfície do solo ou levemente incorporadas apresentam maior taxa de germinação.

Embora seja também uma Euphorbiaceae, a espécie Chamesyce hirta (EPHHI) apresentou comportamento diferenciado em relação à EPHHL, verificando-se que apenas no tratamento 3 sua presença ocorreu nos quatro anos. Nos tratamentos 1, 2, 4, 5, 6 e 7 registrou-se a sua ocorrência, mas com menor constância. Em todas as situações as densidades foram sendo reduzidas a cada ano.

A família Gramineae (Poaceae) foi bem representada nesta pesquisa, pois as espécies ocorrentes estão entre as mais importantes da região. No primeiro ano, o capim-carrapicho (Cenchrus echinatus) esteve presente em todos os tratamentos na segunda avaliação (pósemergência). No segundo ano ele também ocorreu em todos os tratamentos, inclusive na avaliação da época do manejo. No terceiro ano a espécie foi encontrada nos tratamentos 3, 6 e 7. No quarto ano ela foi constatada apenas no tratamento 6 (preparo convencional). Esses resultados indicam que a não-movimentação do solo praticamente eliminou a ocorrência da espécie nas parcelas com plantio direto, independentemente da rotação de culturas utilizada. Considerando os tratamentos 6 (convencional) e 7 (cultivo mínimo), observase que somente no tratamento 6 , com maior movimentação do solo, plantas desta espécie estiveram presentes em todos os anos.

A espécie Brachiaria plantaginea esteve presente somente no primeiro ano nos tratamentos 2, 3, 4 e 7, indicando que o conjunto de fatores, como controle químico, controle cultural, manejo e características inerentes à espécie, proporcionou ambiente desfavorável a ela. Deve ser destacada a elevada eficácia do controle químico empregado na área experimental, com níveis de controle provavelmente superiores aos adotados pelo agricultor em anos anteriores.

Uma das plantas daninhas problemáticas nos sistemas de cultivo mínimo e plantio direto, sobretudo quando se pereniza, é o capimamargoso (Digitaria insularis). Nos dois primeiros anos desta pesquisa ele ocorreu em praticamente todos os tratamentos. No terceiro ano a sua presença foi verificada apenas no cultivo mínimo. No quarto ano, esteve presente nos tratamentos 1 (plantio direto), 6 (preparo convencional) e 7 (cultivo mínimo). Esses resultados permitem concluir que mesmo no sistema de plantio direto é possivel manter plantas daninhas problemáticas sob controle, desde que sejam empregados programas de rotação eficientes combinados com manejo e controle químico adequados. Também pode ser verificado que o fato de se empregar preparo convencional não evita o surgimento de capimamargoso, principalmente se a área estiver próxima ao foco de infestação, pois as sementes são facilmente transportadas pelo vento.

A planta daninha capim-colchão (Digitaria horizontalis) é uma das espécies mais freqüentes nos ambientes agrícolas, principalmente no cultivo convencional. Nesta pesquisa registrouse a sua ocorrência no tratamento 6 (preparo convencional), nos três primeiros anos. Nos tratamentos 1, 3 e 4 ela ocorreu somente no primeiro e no terceiro ano. No tratamento 7 , com cultivo mínimo, registrou-se a presença desta espécie apenas no segundo ano. No quarto ano, esta espécie ocorreu apenas no tratamento 1. Esses resultados indicam que, de maneira geral, a DIGHO não se manteve na área experimental, e a provável justificativa é a mesma apresentada para a Brachiaria plantaginea.

Trabalhos desenvolvidos por Velini \& Martins (1998), Martins et al. (1999) e Velini \& Negrissoli (2000), estudando a dinâmica de populações de plantas daninhas em cana crua com diferentes quantidades de palhada, constataram que, em geral, as espécies de plantas daninhas pertencentes à família Gramineae apresentaram maior sensibilidade aos efeitos da palha. Neste trabalho, essa tendência foi confirmada mesmo utilizando coberturas mortas provenientes de fontes diferentes. 
Para outras espécies não houve um padrão lógico de comportamento. Alternanthera tenella (ALRTE) e Leonotis nepetifolia (LEONE) foram encontradas somente no quarto ano nos sistemas convencional, cultivo mínimo e alguns tratamentos em plantio direto (tratamentos 1 , 3, 4 e 5 para LEONE e 2, 3 e 4 para ALRTE).

A espécie Sida rhombifolia (SIDRH) foi constatada apenas nos tratamentos 6 e 7 (sistemas convencional e mínimo). No terceiro ano ocorreu no tratamento $6 \mathrm{e}$, no quarto, foi detectada nos tratamentos 6 e 7 . Dado o sistema radicular profundo e agressivo, observações de campo têm relacionado a presença freqüente desta planta daninha em solos com elevado grau de compactação. Plantas daninhas que ocorreram somente no primeiro ano da pesquisa podem ter sofrido processos de controle e supressão suficientes para deixar de infestar significativamente a área - é o caso de Portulaca oleraceae (POROL), Spermacoce latifolia (BOILF) e Amaranthus viridis (AMAVI). A trapoeraba (Commelina benghalensis) foi detectada no primeiro e no segundo ano, em três tratamentos em plantio direto e no cultivo mínimo. Já a espécie Ageratum conyzoides foi observada no segundo ano no tratamento 2 e no tratamento 7 e, no terceiro, nos tratamentos 2,4 e 5 (plantio direto), 6 (preparo convencional) e 7 (cultivo mínimo). Os resultados estão apresentados nas Tabelas 3, 4, 5, 6, 7, 8, 9 e 10 .

De maneira geral, ao longo dos anos, as densidades das populações da comunidade infestante foram sendo reduzidas em todos os tratamentos, conforme ilustram as Figuras 1 e 2. A exceção ocorreu com o tratamento 4, que na avaliação de manejo no quarto ano apresentou resultado desproporcional à marcha evolutiva da infestação. O aumento da densidade nesta avaliação foi atribuído à presença da espécie $A$. tenella, que surgiu no último ano na maioria dos tratamentos; no tratamento 4, a sua densidade foi significativamente maior.

Os tratamentos em plantio direto, com os respectivos programas de rotação de culturas, quando comparados aos tratamentos sob preparo convencional e/ou cultivo mínimo, foram significativamente superiores em termos de redução das densidades da flora infestante. Blanco et al. (1994), avaliando diferentes manejos sobre a germinação de plantas daninhas, verificaram que, no tratamento em que a área ficava em pousio e o solo era revolvido com grade de disco após as primeiras chuvas, as características da flora infestante eram: Amaranthus viridis - 1.270 plantas $\mathrm{m}^{-2}$, Brachiaria plantaginea - 225 plantas $\mathrm{m}^{-2}$, Digitaria horizontalis - 1.826 plantas $\mathrm{m}^{-2} \mathrm{e}$ Eleusine indica - 500 plantas $\mathrm{m}^{-2}$. Quando a destruição da massa verde foi realizada com herbicidas, sem revolvimento do solo (plantio direto), os resultados foram: A. viridis 352 plantas $\mathrm{m}^{-2}$, B. plantaginea- 20 plantas $\mathrm{m}^{-2}$, $D$. horizontalis - 1.343 plantas $\mathrm{m}^{-2} \mathrm{e} E$. indica 302 plantas $\mathrm{m}^{-2}$. Em estudo conduzido por Pereira (1996), em Botucatu-SP, foram avaliadas as características da comunidade infestante na cultura da soja e concluiu-se que, comparado ao sistema de plantio convencional, o plantio direto sempre apresentou menor quantidade de plantas daninhas, principalmente as pertencentes à família Gramineae (poaceae).

Deve-se ressaltar que, na escolha de um programa de rotação de culturas, devem ser considerados todos os fatores inerentes à realidade local. Conforme pesquisa desenvolvida por Cardina et al. (1991), os efeitos dos sistemas de plantio sobre a distribuição vertical de sementes de plantas daninhas variaram de acordo com o tipo de solo. De acordo com Boström \& Fogelfors (1999), em pesquisa realizada num clima temperado e úmido, a combinação do sistema de cultivo com as características dos restos culturais e as práticas de manejo das plantas daninhas, possibilitou a redução de doses de herbicidas mantendo-se a eficiência de controle.

Com este trabalho, conclui-se que os programas de rotação de culturas, sob sistema de plantio direto, que apresentaram maior eficiência no controle cultural da comunidade infestante foram: programa B - tratamento 2 (sorgo/ soja - crotalária/milho - milheto/soja - milho safrinha/soja); programa A - tratamento 1 (milho safrinha/soja - girassol/milho - sorgo/ soja - girassol/milho); e programa C - tratamento 3 (guandu/soja - milheto/soja - milho safrinha/milho - girassol/soja). O sistema de plantio direto apresentou maior eficiência no controle cultural das plantas daninhas que os sistemas de cultivo mínimo e preparo convencional, reduzindo o número total de indivíduos e a diversidade da comunidade infestante. 


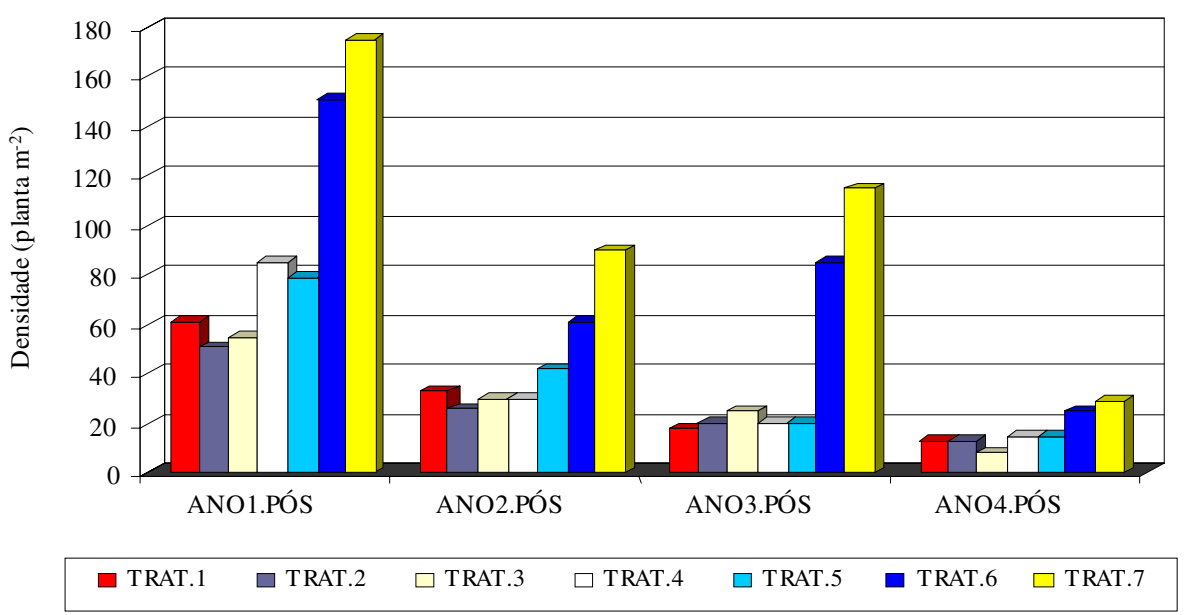

Figura 1 - Dinâmica populacional de plantas daninhas na pós-emergência. Chapadão do Céu-GO. 1994/95 - $1997 / 98$.

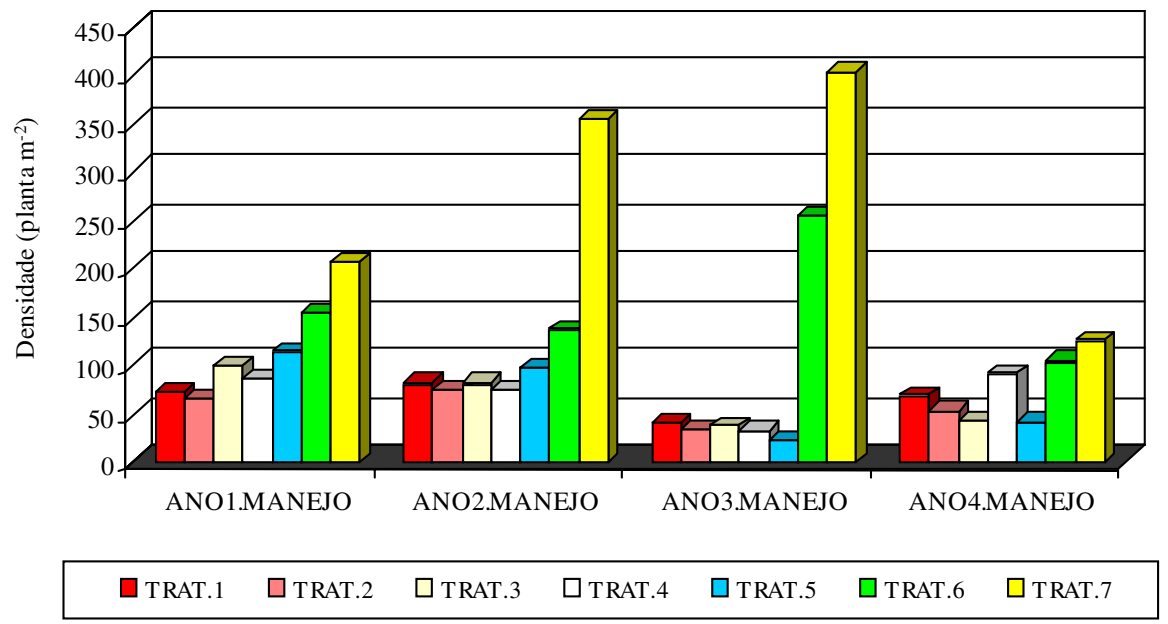

Figura 2 - Dinâmica populacional de plantas daninhas no manejo. Chapadão do Céu-GO. 1994/95 - $1997 / 98$.

Tabela 3 - Avaliações das densidades da comunidade infestante no primeiro ano (1994/95). Chapadão do Céu-GO

\begin{tabular}{|c|c|c|c|c|}
\hline \multirow{2}{*}{ Tratamento } & \multirow{2}{*}{ Rotação } & \multirow{2}{*}{$\begin{array}{c}\text { Sistema } \\
\text { de } \\
\text { cultivo }\end{array}$} & $\begin{array}{c}|c| \\
\text { No } \\
\text { manejo }\end{array}$ & $\begin{array}{c}\text { Na pós- } \\
\text { emergência }\end{array}$ \\
\hline 1 & Programa A & PD & $72 \mathrm{fe}$ & $61 \mathrm{bcd}$ \\
\hline 2 & Programa B & PD & $67 \mathrm{f}$ & $51 \mathrm{~d}$ \\
\hline 3 & Programa C & PD & $101 \mathrm{~cd}$ & $55 \mathrm{~cd}$ \\
\hline 4 & Programa D & PD & $87 \mathrm{de}$ & $85 \mathrm{~b}$ \\
\hline 5 & Programa E & PD & $115 \mathrm{c}$ & $79 \mathrm{bc}$ \\
\hline 6 & Programa F & PC & $155 \mathrm{~b}$ & $151 \mathrm{a}$ \\
\hline 7 & Programa G & CM & $207 \mathrm{a}$ & $175 \mathrm{a}$ \\
\hline CV (\%) & & & 7,28 & 12,57 \\
\hline DMS (5\%) & & & 19,54 & 27,55 \\
\hline
\end{tabular}

Médias abrangidas pela mesma letra não diferem estatisticamente (Tukey 5\%).
Tabela 4 - Avaliações das densidades da comunidade infestante no segundo ano (1995/96). Chapadão do Céu-GO

\begin{tabular}{|c|c|c|c|c|}
\hline \multirow{2}{*}{ Tratamento } & \multirow{2}{*}{ Rotação } & $\begin{array}{c}\text { Sistema } \\
\text { de } \\
\end{array}$ & & \multicolumn{2}{|c|}{ Densidade (plantas m ${ }^{-2}$ ) } \\
\cline { 3 - 5 } & cultivo & $\begin{array}{c}\text { No } \\
\text { manejo }\end{array}$ & $\begin{array}{c}\text { Na pós- } \\
\text { emergência }\end{array}$ \\
\hline 1 & Programa A & PD & $82 \mathrm{c}$ & $45 \mathrm{bc}$ \\
\hline 2 & Programa B & PD & $75 \mathrm{c}$ & $26 \mathrm{c}$ \\
\hline 3 & Programa C & PD & $82 \mathrm{c}$ & $30 \mathrm{c}$ \\
\hline 4 & Programa D & PD & $75 \mathrm{c}$ & $30 \mathrm{c}$ \\
\hline 5 & Programa E & PD & $97 \mathrm{c}$ & $42 \mathrm{bc}$ \\
\hline 6 & Programa F & PC & $138 \mathrm{~b}$ & $61 \mathrm{~b}$ \\
\hline 7 & Programa G & CM & $355 \mathrm{a}$ & $90 \mathrm{a}$ \\
\hline CV (\%) & & & 12,03 & 23,12 \\
\hline DMS (5\%) & & & 36,30 & 25,03 \\
\hline
\end{tabular}

Médias abrangidas pela mesma letra não diferem estatisticamente (Tukey 5\%). 
Tabela 5 - Avaliações das densidades da comunidade infestante no terceiro ano (1996/97). Chapadão do Céu-GO

\begin{tabular}{|c|c|c|c|c|}
\hline \multirow[b]{2}{*}{ Tratamento } & \multirow[b]{2}{*}{ Rotação } & \multirow{2}{*}{$\begin{array}{c}\text { Sistema } \\
\text { de } \\
\text { cultivo }\end{array}$} & \multicolumn{2}{|c|}{ Densidade (plantas $\mathrm{m}^{-2}$ ) } \\
\hline & & & $\begin{array}{c}\text { No } \\
\text { manejo }\end{array}$ & $\begin{array}{c}\text { Na pós- } \\
\text { emergência }\end{array}$ \\
\hline 1 & Programa A & PD & $40 \mathrm{c}$ & $18 \mathrm{c}$ \\
\hline 2 & Programa B & PD & $35 \mathrm{c}$ & $20 \mathrm{c}$ \\
\hline 3 & Programa C & PD & $38 \mathrm{c}$ & $25 \mathrm{c}$ \\
\hline 4 & Programa D & PD & $33 \mathrm{c}$ & $20 \mathrm{c}$ \\
\hline 5 & Programa E & PD & $23 \mathrm{c}$ & $20 \mathrm{c}$ \\
\hline 6 & Programa F & PC & $255 \mathrm{~b}$ & $85 \mathrm{~b}$ \\
\hline 7 & Programa G & $\mathrm{CM}$ & $405 \mathrm{a}$ & $115 \mathrm{a}$ \\
\hline CV $(\%)$ & & & 8,17 & 19,25 \\
\hline $\operatorname{DMS}(5 \%)$ & & & 22,59 & 19,46 \\
\hline
\end{tabular}

Médias abrangidas pela mesma letra não diferem estatisticamente (Tukey 5\%).

Tabela 7 - Espécies e densidades populacionais da comunidade infestante em duas épocas referentes à aplicação de herbicidas: manejo e pós-emergência no primeiro ano. Chapadão do Céu, GO - 1994/95 - 1997/98

\begin{tabular}{|c|c|c|c|c|}
\hline \multirow{2}{*}{ Tratamento } & \multicolumn{2}{|c|}{ Aplicação de manejo } & \multicolumn{2}{|c|}{$\begin{array}{l}\text { Aplicação em pós- } \\
\text { emergência }\end{array}$} \\
\hline & Espécie & 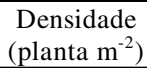 & Espécie & $\begin{array}{l}\text { Densidade } \\
\left(\text { planta }^{-2}\right)\end{array}$ \\
\hline \multirow{4}{*}{1} & BIDPI & 19 & CCHEC & 18 \\
\hline & DIGIN & 25 & BIDPI & 21 \\
\hline & EPHHL & 18 & IAOGR & 12 \\
\hline & IAOGR & 10 & DIGHO & 10 \\
\hline \multirow{4}{*}{2} & BRAPL & 20 & IAOGR & 10 \\
\hline & EPHHI & 15 & BIDPI & 18 \\
\hline & IAOGR & 12 & CCHEC & 15 \\
\hline & "BIDPI & 20 & AMAVI & 08 \\
\hline \multirow{4}{*}{3} & BIDPI & 25 & IDPI & 15 \\
\hline & DIGHO & 41 & AMAVI & 12 \\
\hline & EPHHI & 23 & CCHEC & 18 \\
\hline & BRAPL & 12 & BOILF & 10 \\
\hline \multirow{4}{*}{4} & IAOGR & 15 & IAOGR & 10 \\
\hline & DIGHO & 15 & CCHEC & 25 \\
\hline & BIDPI & 45 & DIGHO & 30 \\
\hline & BRAPL & 12 & BIDPI & 20 \\
\hline \multirow{4}{*}{5} & BIDPI & 28 & $\mathrm{CCHEC}$ & 18 \\
\hline & DIGIN & 42 & BIDPI & 31 \\
\hline & EPHHI & 25 & $\mathrm{COMBE}$ & 10 \\
\hline & AMAVI & 20 & IAOGR & 20 \\
\hline \multirow{4}{*}{6} & CCHEC & 60 & CCHEC & 55 \\
\hline & DIGHO & 30 & BOILF & 40 \\
\hline & EPHHI & 40 & BIDPI & 26 \\
\hline & BIDPI & 25 & DIGHO & 30 \\
\hline \multirow{8}{*}{7} & EPHHL & 20 & BIDPI & 50 \\
\hline & POROL & 35 & DIGIN & 50 \\
\hline & CCHEC & 50 & $\mathrm{COMBE}$ & 30 \\
\hline & COMBE & 15 & CCHEC & 45 \\
\hline & DIGIN & 25 & & \\
\hline & BRAPL & 15 & & \\
\hline & IAOGR & 12 & & \\
\hline & BIDPI & 35 & & \\
\hline
\end{tabular}

Tabela 6 - Avaliações das densidades da comunidade infestante no quarto ano (1997/98). Chapadão do Céu-GO.

\begin{tabular}{|c|c|c|c|c|}
\hline \multirow{2}{*}{ Tratamento } & \multirow{2}{*}{ Rotação } & \multirow{2}{*}{$\begin{array}{c}\text { Sistema } \\
\text { de }\end{array}$} & & \multicolumn{2}{|c|}{ Densidade (plantas $\left.\mathrm{m}^{-2}\right)$} \\
\cline { 4 - 5 } & cultivo & $\begin{array}{c}\text { No } \\
\text { manejo }\end{array}$ & $\begin{array}{c}\text { Na pós- } \\
\text { emergência }\end{array}$ \\
\hline 1 & Programa A & PD & $69 \mathrm{c}$ & $13 \mathrm{~b}$ \\
\hline 2 & Programa B & PD & $53 \mathrm{~cd}$ & $13 \mathrm{~b}$ \\
\hline 3 & Programa C & PD & $43 \mathrm{~d}$ & $8 \mathrm{~b}$ \\
\hline 4 & Programa D & PD & $92 \mathrm{~b}$ & $15 \mathrm{~b}$ \\
\hline 5 & Programa E & PD & $42 \mathrm{~b}$ & $15 \mathrm{~b}$ \\
\hline 6 & Programa F & PC & $105 \mathrm{~b}$ & $25 \mathrm{a}$ \\
\hline 7 & Programa G & CM & $127 \mathrm{a}$ & $29 \mathrm{a}$ \\
\hline CV (\%) & & & 9,10 & 18,09 \\
\hline DMS (5\%) & & & 16,15 & 7,12 \\
\hline
\end{tabular}

Médias abrangidas pela mesma letra não diferem estatisticamente (Tukey 5\%).

Tabela 8 - Espécies e densidades populacionais da comunidade infestante em duas épocas referentes à aplicação de herbicidas: manejo e pós-emergência no segundo ano. Chapadão do Céu-GO, 1994/95 - 1997/98

\begin{tabular}{|c|c|c|c|c|}
\hline \multirow{2}{*}{ Tratamento } & \multicolumn{2}{|c|}{ Aplicação de manejo } & \multicolumn{2}{|c|}{$\begin{array}{c}\text { Aplicação em pós- } \\
\text { emergência }\end{array}$} \\
\hline & Espécie & $\begin{array}{c}\text { Densidade } \\
\left(\text { planta }^{-2}\right)\end{array}$ & Espécie & $\begin{array}{l}\text { Densidade } \\
\left({\left.\text { planta } \mathrm{m}^{-2}\right)}^{\text {a }}\right.\end{array}$ \\
\hline \multirow{4}{*}{1} & BIDPI & 28 & CCHEC & 15 \\
\hline & IAOGR & 12 & IAOGR & 08 \\
\hline & CCHEC & 32 & BIDPI & 10 \\
\hline & "EPHHL & 10 & & \\
\hline \multirow{4}{*}{2} & IAOGR & 12 & BIDPI & 08 \\
\hline & BIDPI & 40 & CCHEC & 10 \\
\hline & CCHEC & 15 & AGECO & 08 \\
\hline & COMBE & 08 & & \\
\hline \multirow{3}{*}{3} & BIDPI & 50 & EPHHL & 05 \\
\hline & IAOGR & 12 & BIDPI & 15 \\
\hline & "EPHHI & 20 & CCHEC & 10 \\
\hline \multirow{4}{*}{4} & COMBE & 15 & BIDPI & 10 \\
\hline & BIDPI & 30 & CCHEC & 15 \\
\hline & CCHEC & 20 & IAOGR & 05 \\
\hline & "EPHHI & 10 & & \\
\hline \multirow{4}{*}{5} & BIDPI & 50 & CCHEC & 15 \\
\hline & CCHEC & 20 & BIDPI & 15 \\
\hline & IAOGR & 12 & EPHHL & 12 \\
\hline & "EPHHL & 15 & & \\
\hline \multirow{4}{*}{6} & BIDPI & 60 & DIGHO & 12 \\
\hline & CCHEC & 41 & BIDPI & 25 \\
\hline & IAOGR & 12 & EPHHL & 12 \\
\hline & DIGHO & 25 & IAOGR & 12 \\
\hline \multirow{8}{*}{7} & CCHEC & 50 & AGECO & 25 \\
\hline & BIDPI & 100 & IAOGR & 20 \\
\hline & IAOGR & 50 & BIDPI & 30 \\
\hline & COMBE & 35 & CCHEC & 15 \\
\hline & DIGIN & 40 & & \\
\hline & DIGHO & 45 & & \\
\hline & EPHHL & 20 & & \\
\hline & ЕРНHI & 15 & & \\
\hline
\end{tabular}


Tabela 9 - Espécies e densidades populacionais da comunidade infestante em duas épocas referentes à aplicação de herbicidas: manejo e pós-emergência no terceiro ano. Chapadão do Céu-GO, 1994/95 - 1997/98

\begin{tabular}{|c|c|c|c|c|}
\hline \multirow{2}{*}{ Tratamento } & \multicolumn{2}{|c|}{ Aplicação de manejo } & \multicolumn{2}{|c|}{$\begin{array}{l}\text { Aplicação em pós- } \\
\text { emergência }\end{array}$} \\
\hline & Espécie & 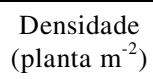 & Espécie & $\begin{array}{l}\text { Densidade } \\
\left(\text { planta }^{-2}\right)\end{array}$ \\
\hline \multirow{3}{*}{1} & DIGIN & 15 & BIDPI & 08 \\
\hline & EPHHI & 10 & EPHHL & 05 \\
\hline & BIDPI & 15 & EPHHI & 05 \\
\hline \multirow{3}{*}{2} & AGECO & 10 & BIDPI & 05 \\
\hline & BIDPI & 15 & DIGHO & 10 \\
\hline & DIGHO & 10 & EPHHI & 05 \\
\hline \multirow{3}{*}{3} & BIDPI & 15 & BIDPI & 10 \\
\hline & EPHHI & 10 & IAOGR & 05 \\
\hline & IAOGR & 08 & CCHEC & 10 \\
\hline \multirow{3}{*}{4} & BIDPI & 10 & BIDPI & 05 \\
\hline & AGECO & 15 & IAOGR & 05 \\
\hline & IAOGR & 08 & DIGHO & 10 \\
\hline \multirow{3}{*}{5} & BIDPI & 08 & BIDPI & 05 \\
\hline & AGECO & 10 & EPHHL & 05 \\
\hline & EPHHI & 05 & AGECO & 10 \\
\hline \multirow{6}{*}{6} & BIDPI & 100 & BIDPI & 30 \\
\hline & SIDRH & 10 & IAOGR & 20 \\
\hline & EPHHI & 25 & CCHEC & 35 \\
\hline & DIGIN & 40 & & \\
\hline & IAOGR & 30 & & \\
\hline & AGECO & 50 & & \\
\hline \multirow{7}{*}{7} & BIDPI & 150 & CCHEC & 40 \\
\hline & DIGIN & 50 & BIDPI & 50 \\
\hline & IAOGR & 30 & AGECO & 10 \\
\hline & AGECO & 100 & EPHHL & 15 \\
\hline & CCHEC & 40 & & \\
\hline & EPHHI & 15 & & \\
\hline & EPHHL & 20 & & \\
\hline
\end{tabular}

\section{LITERATURA CITADA}

ADEGAS, F. S. Manejo integrado de plantas daninhas em plantio direto no Paraná. In: SEMINÁRIO NACIONAL SOBRE MANEJO E CONTROLE DE PLANTAS DANINHAS EM PLANTIO DIRETO, 1., 1998, Passo Fundo-RS. Palestras... Passo Fundo, Aldeia Norte, 1998. p. 17-26.

ALMEIDA, F. S. Effect of some winter crop mulch on soil weed infestation. In: CROP PROTECTION CONFERENCE, 5., 1985, British. Proceedings... British: Weeds, 1985. v. 2, p. 651-659.

Planta Daninha, Viçosa-MG, v.21, n.3, p.355-363, 2003
Tabela 10 - Espécies e densidades populacionais da comunidade infestante em duas épocas referentes à aplicação de herbicidas: manejo e pós-emergência no quarto ano. Chapadão do Céu-GO, 1994/95 - 1997/98

\begin{tabular}{|c|c|c|c|c|}
\hline \multirow{2}{*}{ Tratamento } & \multicolumn{2}{|c|}{ Aplicação de manejo } & \multicolumn{2}{|c|}{$\begin{array}{l}\text { Aplicação em pós- } \\
\text { emergência }\end{array}$} \\
\hline & Espécie & $\begin{array}{l}\text { Densidade } \\
\left(\text { planta }^{-2}\right)\end{array}$ & Espécie & $\begin{array}{l}\text { Densidade } \\
\left({\left.\text { planta } \mathrm{m}^{-2}\right)}^{\text {Dan }}\right.\end{array}$ \\
\hline \multirow{5}{*}{1} & DIGIN & 14 & BIDPI & 06 \\
\hline & EPHHI & 14 & EPHHI & 05 \\
\hline & LEONE & 13 & DIGIN & 02 \\
\hline & DIGHO & 10 & & \\
\hline & BIDPI & 18 & & \\
\hline \multirow{5}{*}{2} & EPHHL & 08 & BIDPI & 04 \\
\hline & BIDPI & 20 & DIGIN & 03 \\
\hline & ALRTE & 12 & EPHHI & 06 \\
\hline & IAOGR & 03 & & \\
\hline & EPHHI & 10 & & \\
\hline \multirow{5}{*}{3} & ALRTE & 14 & BIDPI & 02 \\
\hline & BIDPI & 10 & EPHHI & 04 \\
\hline & LEONE & 08 & DIGIN & 02 \\
\hline & EPHHL & 03 & & \\
\hline & EPHHI & 08 & & \\
\hline \multirow{5}{*}{4} & DIGIN & 05 & BIDPI & 04 \\
\hline & LEONE & 20 & EPHHI & 05 \\
\hline & EPHHI & 14 & EPHHL & 06 \\
\hline & BIDPI & 13 & & \\
\hline & ALRTE & 40 & & \\
\hline \multirow{4}{*}{5} & BIDPI & 14 & BIDPI & 04 \\
\hline & LEONE & 05 & EPHHI & 05 \\
\hline & DIGIN & 12 & DIGIN & 06 \\
\hline & EPHHI & 11 & & \\
\hline \multirow{7}{*}{6} & BIDPI & 55 & BIDPI & 04 \\
\hline & SIDRH & 06 & DIGIN & 09 \\
\hline & EPHHI & 08 & EPHHI & 12 \\
\hline & DIGIN & 10 & & \\
\hline & ALRTE & 12 & & \\
\hline & CCHEC & 08 & & \\
\hline & LEONE & 06 & & \\
\hline \multirow{6}{*}{7} & BIDPI & 44 & EPHHL & 05 \\
\hline & LEONE & 12 & BIDPI & 04 \\
\hline & SIDRH & 18 & ALRTE & 20 \\
\hline & DIGIN & 21 & & \\
\hline & ALRTE & 20 & & \\
\hline & EPHHL & 12 & & \\
\hline
\end{tabular}

BIANCHI, M. A. Manejo integrado de plantas daninhas no sistema plantio direto. In: SEMINÁRIO NACIONAL SOBRE MANEJO E CONTROLE DE PLANTAS DANINHAS EM PLANTIO DIRETO, 1., 1998, Passo Fundo-RS. Palestras... Passo Fundo, Aldeia Norte, 1998. p. 108-118.

BLANCO, H. G.; AREVALO, R. A.; BLANCO, F. M. G. Distribuição mensal da emergência de seis ervas daninhas em solos com e sem cultivos. Planta Daninha, v. 12, n. 2 , p. 78-83, 1994. 
BOSTRÖM, U.; FOGELFORS, H. Type and time of autumn tillage with and without herbicides at reduced rates in southern Sweden - Weed flora and diversity. Soil \& Tillage Research, v. 50, p. 283-293, 1999.

CARDINA, J.; REGNIER, E.; HARRISON, K. Long-term tillge effects on seed banks in three Ohio soils. Weed Science, v. 39, p. 186-194, 1991.

LORENZI, H. Manual de identificação e controle de plantas daninhas: plantio direto e convencional. 5.ed. Nova Odessa: Instituto Plantarum, 2000. 339 p.

MARTINS, D.; VELINI, E. D.; MARTINS, C. C.; SOUZA, L. S. Emergência em campo de dicotiledôneas infestantes em solo coberto com palha de cana-de-açúcar. Planta Daninha, v. 17, n.1, p. 151-161, 1999.

PEREIRA, E. S. Avaliações qualitativas e quantitavas das plantas daninhas na cultura da soja (Glycine max (L.) Merril) submetidas aos sistemas de plantio direto e convencional. 1996. 84 f. Dissertação (Mestrado em Agricultura) - Universidade Estadual de São Paulo, Botucatu, 1996.
RUEDELL, J. Plantio direto na região de Cruz Alta. Cruz Alta: FUNDACEP/BASF, 1995. 134 p.

VARGAS, L.; DIAS, D. C.; SILVA, A. A.; ESCHER, V. Emergência de plântulas de leiteiro (Euphorbia heterophylla L.) resistentes e sensíveis aos inibidores de als, em diferentes profundidades de semeadura. Ciência das Plantas Daninhas, v. 5, n. 1, p. 8, 1999.

VELINI, E. D.; MARTINS, D. Efeito da palha de canade-açúcar sobre a germinação das principais espécies de plantas daninhas desta cultura. Botucatu: Universidade Estadual de São Paulo, 1998. 26 p. (Relatório Técnico)

VELINI, E. D.; NEGRISSOLI, E. Controle de plantas daninhas em cana crua. In: CONGRESSO BRASILEIRO DA CIÊNCIA DE PLANTAS DANINHAS, 22., 2000, Foz do Iguaçu. Palestras... Londrina: Sociedade Brasileira da Ciência das Plantas Daninhas, 2000. p. $148-164$. 